\title{
Blockage of RelB expression by gene silencing enhances the radiosensitivity of androgen-independent prostate cancer cells
}

\author{
HENG-CHENG ZHU, TAO QIU, CHAO DAN, XIU-HENG LIU and CHUN-HAI HU \\ Department of Urology, Renmin Hospital of Wuhan University, Wuhan, Hubei 430060, P.R. China
}

Received March 21, 2014; Accepted August 29, 2014

DOI: $10.3892 / \mathrm{mmr} .2014 .2857$

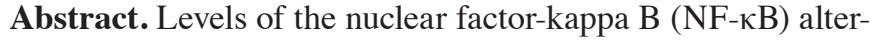
native pathway member RelB have been shown to correlate with the effect of radiation therapy in prostate cancer. RelB expression was evaluated by immunohistochemistry in normal prostate, benign prostate hyperplasia and prostate cancer specimens. RM-1 cells were pretreated with RelB siRNA prior to radiation therapy, and RelB expression in cytoplasmic and nuclear extracts was detected by real-time polymerase chain reaction and western blot analysis. The apoptotic rates of experimental RM-1 cell groups were assessed by flow cytometry. A clonogenic growth array was used to evaluate the radiosensitivity of RM-1 cell groups. The NF- $\mathrm{RB}$ family member RelB was expressed at a high level in prostate cancer specimens. Compared with irradiated control cells, RM-1 cells transfected with RelB siRNA and treated with radiation therapy demonstrated a significant downregulation of RelB expression in the cytoplasm and nucleus. Notably, flow cytometry revealed that pretreatment of RM-1 cells with RelB siRNA enhanced the apoptotic rate in response to radiation therapy compared with controls. Clonogenic growth assay results revealed enhanced radiosensitivity of RelB siRNA cells at various dosage points compared with control groups. Blockage of the alternative $\mathrm{NF}-\kappa \mathrm{B}$ pathway via RelB silencing is a promising approach to enhance the radiosensitivity of prostate cancer.
\end{abstract}

\section{Introduction}

In North America, prostate cancer is currently the most commonly diagnosed noncutaneous cancer in males (1). Its incidence and mortality rate are increasing in China (2). Although the majority of males with prostate cancer may be successfully treated with surgery or radiation therapy, $20-40 \%$ of cases will biochemically recur within 10 years of

Correspondence to: Dr Tao Qiu, Department of Urology, Renmin Hospital of Wuhan University, 238 JieFang Road, Wuhan, Hubei 430060, P.R. China

E-mail: mdqiutao@yahoo.com

Key words: RelB, RNA interference, prostate cancer, radiosensitivity treatment. This risk of recurrence is elevated to $\sim 50 \%$ in males with locally advanced disease, a condition that is primarily managed by radiation therapy. Thus, new technologies that improve the therapeutic index of radiation therapy for local disease have the opportunity to significantly impact on the morbidity and mortality rates of prostate cancer (3).

Higher doses of radiation may be used to improve the outcome of radiation therapy. When high doses (>7,200 cGy) of radiation therapy were applied, the 5-year survival rate increased from 41 to $75 \%$ in patients with unfavorable tumors, including those with T3-stage lesions, initial prostate-specific antigen levels greater than $10 \mathrm{ng} / \mathrm{ml}$, or a biopsy Gleason score greater than 7 (4). However, the use of high-dose radiation treatment is limited due to serious side effects and late complications, including erectile dysfunction, bladder inflammation, urethral stricture and radiation proctitis, caused by a high dose of radiation toxicity (5). Moreover, hormone-independent prostate cancer is resistant to radiation therapy compared with hormone-dependent prostate cancer. Therefore, it is critical to increase the radiation sensitivity of prostate cancer cells to minimize the radiation toxicity by lowering effective therapeutic doses and increase the radiation therapy benefit.

Nuclear factor-kappa B $(\mathrm{NF}-\kappa \mathrm{B})$ proteins are a group of transcriptional regulators that activate the expression of genes involved in diverse biological processes, including inflammation and cell growth, differentiation and apoptosis (6). Activation of $\mathrm{NF}-\kappa \mathrm{B}$ signaling is considered to be a significant mechanism in the development of prostate cancer. Insights obtained from the study of $\mathrm{NF}-\kappa \mathrm{B}$ functions in cancer have provided a mechanistic link between inflammation and tumorigenesis, as well as valuable information regarding $\mathrm{NF}-\kappa \mathrm{B}-$ mediated cytoprotection against cancer therapeutics. Consequently, $\mathrm{NF}-\kappa \mathrm{B}$ is being considered as a target for anticancer therapy $(7,8)$. All subunits of the NF- $\mathrm{B}$ family, RelA, p50, RelB, p52 and c-Rel, are expressed in prostate tissues, and all but c-Rel are detectable in the nucleus of prostatic cells as well as other cancerous tissues (5). This suggests that both the canonical (RelA and p50) and non-canonical (RelB and p52) NF- $\kappa$ B subunits may be activated in prostate cancer cells, whereas c-Rel remains inactive (9). Upon radiation, the $\mathrm{NF}-\kappa \mathrm{B}$ pathway is activated by radiation-induced reactive oxygen species $(9,10)$. The level of nuclear RelB has been shown to correlate with prostate cancer patient Gleason scores, suggesting that RelB may be associated with prostate cancer progression. In numerous types of cancer, the RelA-based classic pathway is considered 
to play a significant role in response to chemotherapeutics and radiotherapeutics (9). However, in prostate cancer, RelB may contribute to the radioresistance of high-risk prostate cancers (11). Therefore, targeting RelB activation may prove to be a valid strategy to defeat aggressive and radiation-resistant prostate cancer.

In the present study, it was demonstrated that RelB inhibition by specific RelB siRNA prior to radiotherapy significantly increased the radiation sensitivity of RM-1 cells.

\section{Materials and methods}

RelB expression in prostate samples. Specimens were acquired from tissue from radical prostatectomy surgery and transurethral resection of the prostate, and normal human prostate was acquired from donors following cardiac mortality. The 71 prostate samples were collected between January 2012 and December 2013 at Renmin Hospital of Wuhan Univeristy (Wuhan, China). The age range of the patients was between 45 and 77 years old. All the specimens were collected following the provision of informed consent from the patient and their family. The study was approved by the ethics committee of Renmin Hospital of Wuhan University, China. Paraffin-embedded sections of normal human prostate $(n=18)$, benign prostate hyperplasia $(\mathrm{BPH} ; \mathrm{n}=29)$ and prostate cancer ( $n=24$; T1cN0M0-T3a N0M0) were obtained from Renmin Hospital of Wuhan University. The prostate cancer specimens were from the androgen-independent stage of cancer. The specimens were stained with rabbit anti-human RelB monoclonal antibody (Santa Cruz Biotechnology, Inc., Santa Cruz, CA, USA) and developed with 3,3-diaminobenzidine. All slides were reviewed by one pathologist who was blinded to the study. Samples were scored based on RelB expression intensity as 0 (negative), 1 (weak), 2 (moderate) or 3 (strong).

Cell culture. The mouse prostate cancer cell line RM-1, a highly aggressive and androgen-independent murine prostatic cancer cell line, was purchased from the Chinese Academy of Sciences (Beijing, China) and maintained in Dulbecco's modified Eagle's medium (Hyclone, Logan, UT, USA) supplemented with $10 \%$ fetal bovine serum, $2 \mathrm{mM}$ L-glutamine, $100 \mathrm{U} / \mathrm{ml}$ penicillin and $100 \mu \mathrm{g} / \mathrm{ml}$ streptomycin, at $37^{\circ} \mathrm{C}$ in a humidified atmosphere containing $5 \% \mathrm{CO}_{2}$.

Construction of recombinant lentivirus vectors. Five types of shRNA sequence targeting the mRNA sequence of RelB (accession number, M83380) were designed and evaluated for specificity and knockdown efficiency. The most effective shRNA for RelB targeting was 5'-TGTCGTCAGGATCT GCTTCTTCAAGAGAGAAGCAGATCCTGACGACATTTT TTC-3' and 3'-ACAGCAGTCCTAGACGAAGAAGTTCTCTC TTCGTCTAGGACTGCTGTAAAAAAGAGCT-5'. shRNA was designed, chemically synthesized and purified by polyacrylamide gel electrophoresis (PAGE) in the absence of RNase contamination, according to the manufacturer's instructions (R\&S, Shanghai, China). The lentiviral work vector plentilox3.7 was used to recombine the shuttle plasmid. The shRNAs were cloned to plentilox3.7, which was linearized by restriction endonucleases HindIII and KspAI. All constructs were verified by sequencing. The recombinant lentiviral vectors containing RelB-specific siRNA were designated as Lenti-siRNA-RelB. A vector containing siRNA targeting luciferase, designated as Lenti-siRNA, was used as a control. To produce the lentivirus, the recombinant work vector and package plasmids (pVSVG, pRSV-REV and pMDLg/pRRE) were cotransfected to $293 \mathrm{~T}$ cells using Lipofectamine 2000 (Invitrogen Life Technologies, Carlsbad, CA, USA). The culture medium was collected $48 \mathrm{~h}$ later, concentrated by ultracentrifugation, aliquoted and stored at $-80^{\circ} \mathrm{C}$ for later use. Vector titers were determined by measuring GFP expression in $293 \mathrm{~T}$ cells transduced with serial dilutions of vectors, and transducing units (TU)/ml were calculated as the number of cells infected, as follows: percentage of $\mathrm{EGFP}^{+}$cells / $10 \mathrm{x}$ dilution of vector. The vector titers were $1 \times 10^{8} \mathrm{TU} / \mathrm{ml}$. The RM-1 cells were transfected at a multiplicity of infection (MOI) of 100. Based on the results of fluorescence microscopy and flow cytometry, the infection efficiency reaches up to $95 \%$ with an MOI of 100 (12).

Radiation treatment of RM-1 cells. The cells were divided into four groups for the radiation therapy analysis. In the control group, RM-1 cells were treated with phosphate-buffered saline (PBS). In the radiation therapy group (RT group), RM-1 cells were treated with PBS and exposed to radiation at a single dose of 6 Gy. In the siRNA luciferase and radiation therapy group (siRNA control+RT group), RM-1 cells were transfected with siRNA targeting luciferase at an MOI of 100 and, after $72 \mathrm{~h}$, cells were exposed to radiation at a single dose of $6 \mathrm{~Gy}$. In the siRNA RelB and radiation therapy group (siRNA RelB+RT group), RM-1 cells were transfected with siRNA RelB at an MOI of 100 , and after $72 \mathrm{~h}$ cells were exposed to radiation at a single dose of $6 \mathrm{~Gy}$.

RM-1 cells in six-well plates were irradiated using a 6-MeV linear accelerator at room temperature. Doses were administered at $2.25 \mathrm{~Gy} / \mathrm{min}$. The distance between the radiation source and the cells was $100 \mathrm{~cm}$. Following radiation therapy, the cells were incubated in normal medium for $48 \mathrm{~h}$ and used for subsequent experiments. In the clonogenic survival assay, each cell group received a single dose of $0,2,4,6$ or 8 Gy per treatment following infection with lentivirus.

Real-time polymerase chain reaction (PCR). Total RNA $(1 \mu \mathrm{g})$ was isolated from RM-1 cells with TRIzol reagent and reverse-transcribed using oligo-dT primers with reverse transcription reagents (Toyobo Corporation, Osaka, Japan). Reverse-transcribed RNA was amplified with SYBR-Green PCR Master mix (Toyobo Corporation) plus $0.4 \mu \mathrm{M}$ gene-specific upstream and downstream primers on a 7500 Fast Realtime cycler (Applied Biosystems, Inc., Foster City, CA, USA). PCR conditions were as follows: 40 cycles of denaturation at $95^{\circ} \mathrm{C}$ for $15 \mathrm{sec}$, annealing at $55^{\circ} \mathrm{C}$ for $15 \mathrm{sec}$, and extension at $72^{\circ} \mathrm{C}$ for $45 \mathrm{sec}$. The primer sequences were as follows: Forward, CCGTGAAAAGATGACCCAG and reverse, TAGCCACGCTCGGTCAGG for $\beta$-actin; forward, GACATACCGTGGTGTTCAGC and reverse, GTCCTCGTAGGGTGGCGTT for RelB. Data were analyzed by relative quantification using the $2^{-\Delta \Delta \mathrm{CT}}$ method (13).

Western blot analysis. Proteins from the cytosol and nucleus were isolated from RM-1 cells. Protein samples were separated 
A

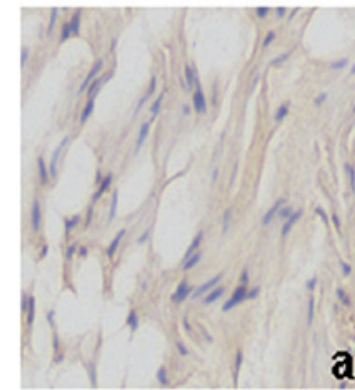

Normal prostate

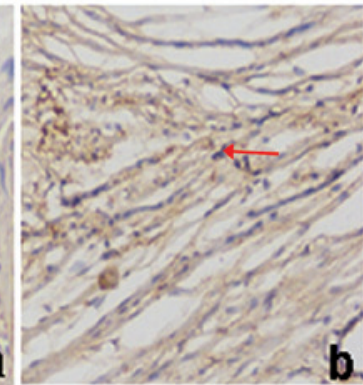

$\mathrm{BPH}$

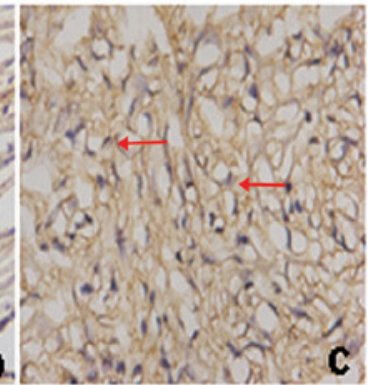

$\mathrm{PCa}$
B

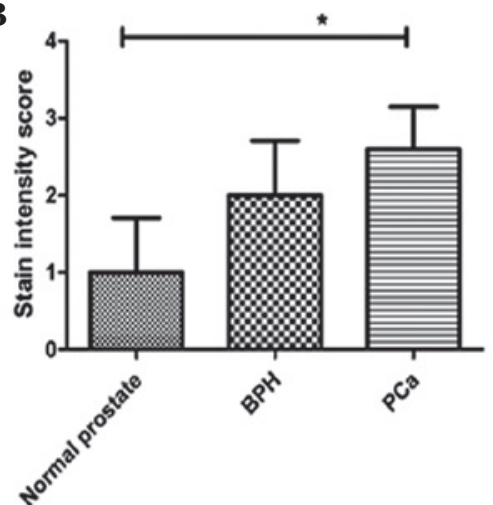

Figure 1. RelB expression is upregulated in prostate cancer. (A) Immunohistochemical analysis of RelB expression in normal prostate, benign prostate hyper-

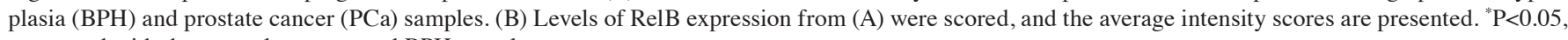
compared with the normal prostate and $\mathrm{BPH}$ samples.

using 10\% SDS-PAGE and transferred onto a nitrocellulose membrane.RelB protein was detected with a primary rabbit anti-mouse RelB monoclonal antibody (1:1,000; Invitrogen Life Technologies) and a horseradish peroxidase-conjugated goat anti-rabbit secondary polyclonal antibody (KPL, Gaithersburg, MD, USA). The protein signals were visualized with an enhanced chemiluminescence reaction system. $\beta$-actin (Santa Cruz Biotechnology, Inc.) was used as an internal control.

DNA binding ability of $N F-\kappa B$ subunits. The DNA-binding capacity of NF- $\mathrm{B}$ subunits in RM-1 nuclear extracts was measured using the Nuclear Extract ${ }^{\mathrm{TM}}$ and TransAM ${ }^{\mathrm{TM}} \mathrm{NF}-\kappa \mathrm{B}$ kits (Active Motif, Carlsbad, CA, USA) according to the manufacturer's instructions. Briefly, an oligonucleotide containing the NF- $\kappa \mathrm{B}$ consensus binding site (5'-GGGACTTTCC-3') was immobilized in 96-well plates, and nuclear extracts containing activated $\mathrm{NF}-\kappa \mathrm{B}$ were added. Binding of $\mathrm{NF}-\kappa \mathrm{B}$ to the oligonucleotides was detected using a rabbit anti-mouse primary monoclonal antibody (Invitrogen Life Technologies), which accesses an epitope on NF- $\kappa$ B subunits when NF- $\kappa$ B binds its target DNA. The addition of secondary goat anti-rabbit polyclonal antibody (KPL) conjugated to horseradish peroxidase was used to detect the $\mathrm{NF}-\kappa \mathrm{B}$. The specificity of this assay was confirmed by incubations in the presence of an excess of non-immobilized consensus oligonucleotide as a competitor, and blank controls, in which PBS replaced the nuclear extracts. 3,3',5,5'-Tetramethylbenzidine was added, and absorbance was detected at $655 \mathrm{~nm}$.

Measurement of apoptosis by flow cytometry. RM-1 cells were collected, resuspended in binding buffer and stained with Annexin V-FITC and propidium iodide (Nanjing KeyGen Biotech Co., Ltd., Nanjing, China). After washing the samples three times in PBS, the cells were analyzed by flow cytometry.

Clonogenic survival assay. Following radiation exposure, RM-1 cells from each well of the six-well plates were trypsinized and grown in triplicate in 60-mm culture dishes with various densities for 14 days. After the cells were fixed in methanol/acetic acid (3:1) for $30 \mathrm{~min}$, cell clones were counted under a microscope (>50 cells/clone). Plating efficiency (PE) was calculated by dividing the average number of colonies per plate by the amount of cells plated, and then multiplying by 100 . Survival fractions (SFs) were calculated using the following formula: SF = colony number / (cell number cultured x PE). A one-hit multi-target model was fitted to the cell survival curve $\left[\mathrm{SF}=1-1\left(1-\mathrm{e}^{\mathrm{KD}}\right)^{\mathrm{N}}, \mathrm{SF}=1-\left(1-\mathrm{e}^{-\mathrm{D} / \mathrm{D}_{0}}\right)^{\mathrm{N}}\left(\mathrm{D}_{0}=1 / \mathrm{K}\right)\right]$ to determine the quasi-threshold dose $\left(\mathrm{D}_{\mathrm{q}}\right)$, mean lethal dose $\left(\mathrm{D}_{0}\right), 2$ Gy survival fraction $\left(\mathrm{SF}_{2}\right), \mathrm{N}$ value and sensitization enhancement ratio.

Statistical analysis. Statistical analysis was performed using one-way analysis of variance for multiple group comparison using SPSS 11.5 software (SPSS, Inc., Chicago, IL, USA). Data are presented as the mean \pm standard deviation. $\mathrm{P}<0.05$ was considered to indicate a statistically significant difference.

\section{Results}

RelB expression is upregulated in prostate cancer. Paraffin-embedded sections of normal human prostate $(n=18)$, BPH $(n=29)$ and prostate cancer $(n=24)$ were examined and scored for RelB expression levels. All prostate cancer specimens, which were from the androgen-independent stage of cancer, revealed an upregulation of RelB compared with $\mathrm{BPH}$ and normal prostate tissue. The average score of RelB expression in prostate cancer samples was significantly higher than that in normal prostate and BPH (Fig. 1). This finding is consistent with previous studies (14).

siRNA RelB suppresses activation of $N F-\kappa B$ upon radiation of $R M-1$ cells. To more closely examine the required function and mechanisms of RelB in cells, RM-1 cells were first transfected with the appropriate siRNAs and then treated with radiation therapy. As shown in Fig. 2A, the mRNA expression of RelB in the siRNA RelB+RT group was reduced by $\sim 40 \%$ compared with the siRNA control+RT group and the RT group (t-test; $\mathrm{P}<0.05)$.

To further examine the reduced RelB expression in the siRNA RelB+RT group, western blot analysis was performed on the cytosolic and nuclear cell fractions. The results revealed that RelB protein levels were $71.8 \%$ lower in the cytosolic fractions (Fig. 2B) and 70.4\% lower in the nuclear fractions 
A
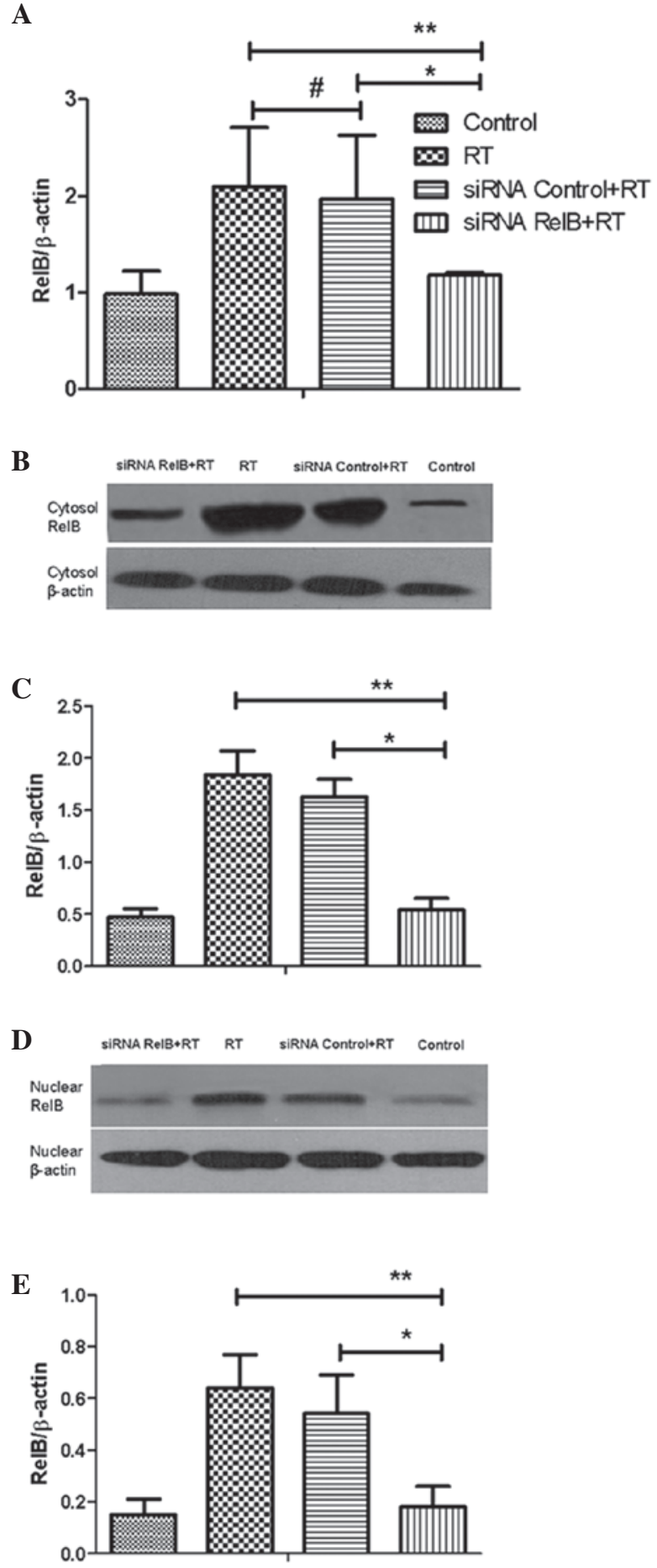

Figure 2. RelB siRNA decreases RelB gene expression and protein levels in RM-1 cells following radiation treatment. (A) RelB mRNA levels in control and RelB siRNA cells treated with radiation. Control, siRNA control and siRNA RelB cells were treated with radiation therapy (RT) using 6 Gy irradiation. Untreated cells were used for comparison. Total RNA was isolated and RelB levels were evaluated by real-time reverse transcription polymerase chain reaction. $\beta$-actin mRNA was used for normalization. (B) RelB protein levels in cytosolic fractions from RM-1 cells transfected with control or RelB siRNA and treated with 6 Gy irradiation. (C) Quantification of RelB protein cytosolic levels from (B). Levels were normalized using $\beta$-actin. RelB cytosolic expression was reduced by $71.8 \%$ in siRNA RelB cells treated with irradiation compared with irradiated siRNA control cells. (D) RelB protein levels in cytosolic fractions from RM-1 cells transfected with control or RelB siRNA and treated with 6 Gy irradiation. (E) Quantification of RelB protein cytosolic levels from (D). Levels were normalized using $\beta$-actin. RelB nuclear expression was reduced by $70.4 \%$ in siRNA RelB cells treated with irradiation compared with irradiated siRNA control cells. ${ }^{* * *} \mathrm{P}<0.05$ and ${ }^{\#} \mathrm{P}>0.05$. Results were obtained from three independent experiments.

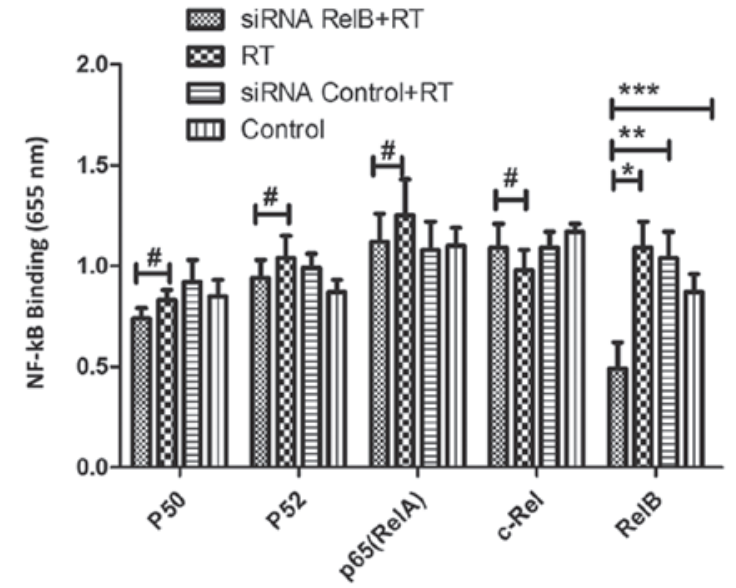

Figure 3. RelB siRNA reduces RelB DNA binding. No significant difference was observed in the DNA binding activity of p50, p52, RelA (p65) and c-Rel in RM-1 cells transfected with siRNA control or RelB and exposed to irradiation. RelB siRNA significantly inhibited RelB DNA binding in the siRNA RelB+RT group compared with the other three groups. Data were obtained from three independent experiments and are expressed as mean \pm SD from three replicates. ${ }^{* * * * * * * *} \mathrm{P}<0.05$ and ${ }^{*} \mathrm{P}>0.05$. RT, radiation therapy.

(Fig. 2D) in the siRNA RelB+RT group compared with those of the siRNA control+RT group, which had similar RelB protein levels to the RT group.

This finding demonstrated that RelB siRNA effectively inhibited the induction of RelB expression in RM-1 cells in response to radiation therapy.

RelB siRNA reduces RelB DNA binding. Next, the DNA-binding ability of NF- $\mathrm{BB}$ subunits was measured in RM-1 nuclear extracts (Fig. 3). No significant differences in the binding abilities of p50, p52, RelA (p65) or c-Rel were observed among the four treated cell groups. However, there was a marked decrease in the RelB DNA binding capacity in the siRNA RelB+RT group. These results revealed that RelB siRNA transfection reduced RelB binding to DNA, but had no effect on the DNA binding activity of p50, p52, RelA (p65) and c-Rel.

RelB siRNA augments radiation therapy-induced apoptosis in $R M-1$ cells. Next, the effects of radiation therapy on inducing apoptosis were examined in the various treated cell groups. As shown in Fig. 4, the siRNA control+RT group and the RT group demonstrated increased apoptosis in response to radiation compared with untreated cells. Notably, the siRNA RelB+RT group had a notably higher apoptotic rate than the siRNA control+RT group and the RT group $(\mathrm{P}<0.05)$. This indicates that silencing of RelB by siRNA increases the levels of apoptosis induced by radiation therapy.

Pretreatment with RelB siRNA increases the radiosensitivity of RM-1 cells. Next, the radiosensitivity of the RM-1 experimental cell groups treated with varying Gy dosages was examined. As shown in Fig. 5, the surviving fraction values from the siRNA RelB+RT group $(0.8,0.11,0.06$ and 0.02 ) were less than those of the siRNA control+RT group $(0.95,0.8,0.12$ and 0.09$)$ at dosages of $2,4,6$ and 8 Gy, indicating that RM-1 cells transfected with RelB siRNA were 
A

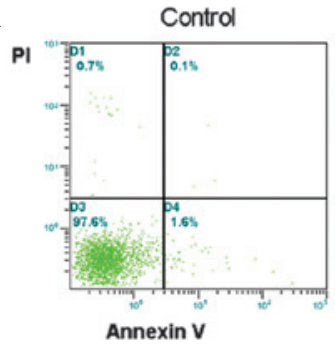

B

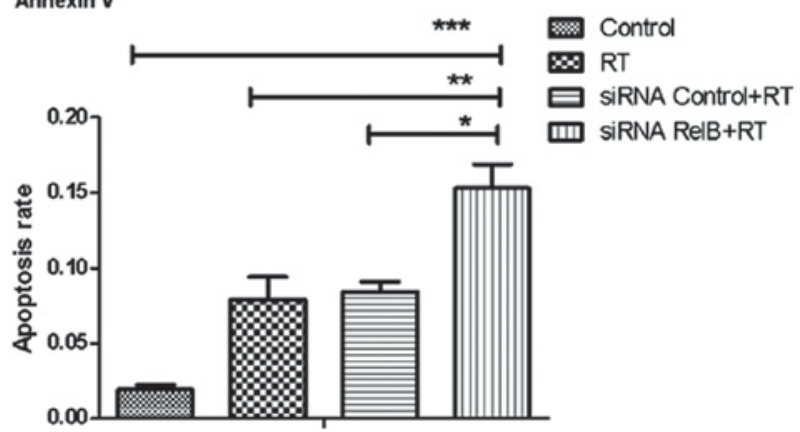

SiRNA Control+RT
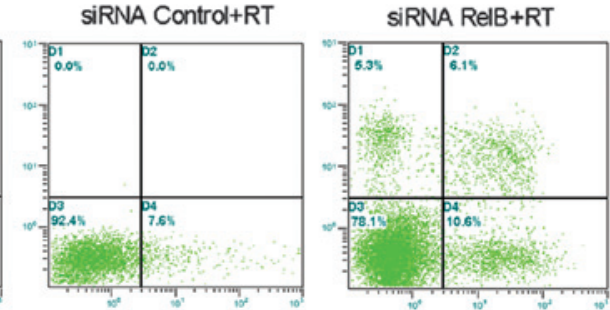

Figure 4. RelB siRNA augments radiation therapy-induced apoptosis in RM-1 cells. (A) Apoptosis was measured using flow cytometry in various RM-1 cell groups treated with 6 Gy, along with untreated RM-1 cells. The image demonstrates typical flow cytometry in the four experimental groups. (B) There was a significant increase in the apoptotic rate of the siRNA RelB and radiation therapy (siRNA RelB+RT) group compared with the other three groups $(*, * * * * * \mathrm{P}<0.05)$. Results were obtained from three independent experiments. $\mathrm{RT}$, radiation therapy.

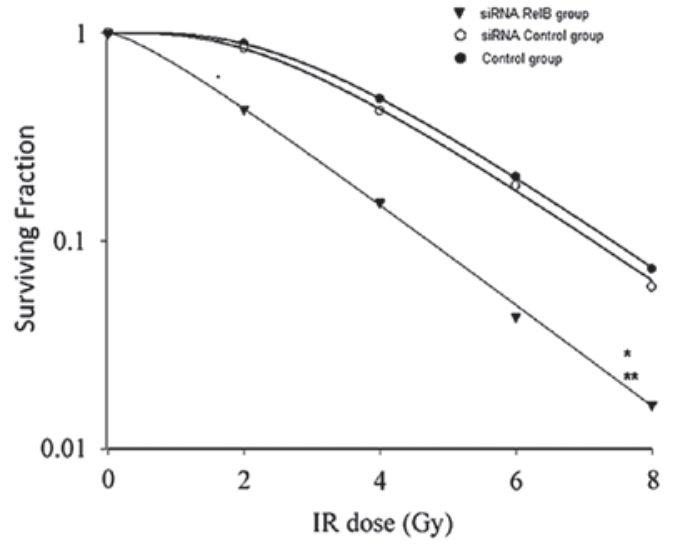

Figure 5. Effect of siRNA RelB on the clonogenic survival of RM-1 cells following radiation therapy. Cells transfected with lentivector were irradiated with a single dose of $0,2,4,6$ or 8 Gy of ionizing radiation (IR). Non-transfected cells were used as controls. Compared with the controls, the siRNA RelB-RM-1 cells were more sensitive to irradiation. ${ }^{*},{ }^{* *} \mathrm{P}<0.05$.

more sensitive to radiation-induced cell death compared with controls.

An increased $\mathrm{D}_{0}$ value indicates a higher cell radiation resistance, while the Dq value represents the cell's ability to recover from sub-lethal damage. The values of $\mathrm{D}_{0}(1.68)$ and Dq (0.60) of the siRNA RelB+RT group were significantly lower than those of the RT group $(1.02,3.08,0.89,4.97)$ and siRNA control+RT group (1.93, 2.76, 0.84, 4.17), indicating that RM-1 cells transfected with RelB siRNA have a lower radiation resistance and a weakened damage recovery ability.

\section{Discussion}

Radiation therapy is in the front-line for the treatment of localized prostate cancer. However, a significant percentage of patients have radiation-resistant disease (15). Cellular hallmarks of cancer include self-sufficiency in growth, loss of growth inhibitory mechanisms, evasion of apoptosis, sustained angiogenesis, immortality and invasion/metastasis (16). All of these mechanisms are known to be affected in some way via the activation of $\mathrm{NF}-\kappa \mathrm{B}$ signaling $(14,17)$.

Traditional research on the mechanisms underlying the effects of radiation has only focused on intracellular damage and repair processes with the influence of radiation treatment on the cellular microenvironment (18). Recently, standard concepts of radiobiology have been based on the assumption that double-strand DNA breaks are the most significant aspects of tumor cell death via free radical formation (19). Studies in radiation biology have acquired new insights in understanding the effects of ionizing radiation on various levels, including immune inflammatory reactions, transmembrane signaling pathways, genomic instability and apoptosis (20). Cellular signaling in response to ionizing radiation involves the complex modulation of various proteins and compensatory stimuli that have not yet been fully characterized (21). Certain findings have revealed that these survival signaling mechanisms have provided cells with a cytoprotective response capability, which allows cells to accelerate proliferation, improve repair functions and resist apoptosis after being exposed to ionizing radiation (22-23). In the setting of clinically relevant doses of ionizing radiation with fractionated schedules, there has been concern that prostate cancer cells may be able to adapt to repeated stressful exposures, possibly resulting in the evolution of a more aggressive phenotype.

A number of studies have reported altered expression of $\mathrm{NF}-\kappa \mathrm{B}$-related proteins in human cancers (24-26). Several studies have shown that the $\mathrm{NF}-\kappa \mathrm{B}$ pathway is a significant factor in radiation resistance, and the classical (canonical) pathway is believed to confer protection of prostate cancer cells from ionizing radiation. Although inhibition of the 
classic pathway may be a useful approach for the enhancement of radiotherapies and chemotherapies, it may also sensitize normal cells to treatment with radiotherapies and chemotherapies.

It is well recognized that $\mathrm{NF}-\kappa \mathrm{B}$ activity promotes radiation resistance, although it is uncertain which $N F-\kappa B$ family member functions in the radiation resistance. Studies on the classic pathway have revealed that suppression of p65 and ablation of IKK $\beta$ increased the chemosensitivity and radiosensitivity of certain cancer cells. However, as the classic pathway is also known to play a significant role in protecting normal tissues against chemotherapies and radiotherapies (11), the overall benefits from suppression of RelA in the sensitization of cancer treatments remain to be investigated.

The alternative pathway, which is involved in prostate cancer aggressiveness, has also been shown to be significant in radiation resistance in prostate cancer (11). The alternative $\mathrm{NF}-\kappa \mathrm{B}$ pathway component RelB protects prostate cancer cells from the detrimental effects of ionizing radiation in part by stimulating expression of the mitochondria-localized antioxidant enzyme manganese superoxide dismutase (MnSOD). Studies have demonstrated that RelB is uniquely expressed at a high level in prostate cancer (Fig. 1) and correlates with high Gleason scores $(11,31)$. Blocking RelB activation suppresses MnSOD expression and sensitizes prostate cancer cells to radiation (15). In the present study, pretreatment with RelB siRNA inhibited RelB expression in the cytosol and nucleus even upon stimulation of radiotherapy (Fig. 2), confirming the blocking of the alternative pathway.

The RelB expression level was shown to be increased in radioresistant $\mathrm{PC}-3$ cells, even though all five subunits of $\mathrm{NF}-\kappa \mathrm{B}$ were expressed in PC-3 cells not treated with radiation (28). Upon pretreatment with the RelB inhibitor $1 \alpha, 25$-dihyroxyvitamin $\mathrm{D}_{3}, \mathrm{PC}-3$ cells remained radiosensitive following traditional treatment (29). Therefore, valid methods to inhibit the alternative pathway needed to be further developed. A further study indicated that ionizing radiation enhanced the DNA binding activity of RelB more than that of RelA in PC-3 cells, indicating that increased RelB nuclear import may be significant in radioresistance development in prostate cancer cells (30). In the current study, the DNA binding activity of RelB was inhibited in the siRNA RelB+RT group, but the binding of other subunits was not affected (Fig. 3). Together, this suggests that the sensitization of prostate cancer to radiotherapy is increased by RelB gene silencing via the inhibition of RelB nuclear import and the reduction of RelB DNA binding activity.

Inhibiting RelB in aggressive androgen-independent PC-3 cells significantly reduces the incidence and growth rate of tumors. One study demonstrated that stable expression of RelB in androgen-responsive LNCaP tumors increased the circulating IL-8 levels. The decrease in tumorigenicity coincided with a reduction in the NF- $\kappa \mathrm{B}$ target interleukin- 8 (IL-8). Consistent with this finding, downregulation of RelB via siRNA also reduced tumor growth and decreased levels of IL-8 (31). The blockage of p50:RelA dimer nuclear translocation by ablating expression or SN50-mediated inhibition of IKK $\beta$ significantly suppressed tumorigenesis and improved therapeutic efficacy in several cancer types (29). The prevention of $\mathrm{p} 52:$ RelB nuclear translocation by expression of $\mathrm{p} 100 \mathrm{M}$ and treatment with SN52 and 1 $\alpha, 25$-dihydroxyvitamin D3 dramatically enhanced the radiosensitization effect $(15,29,30)$. The prevention of RelB nuclear translocation decreased the tumorigenicity of prostate cancer cells. Together, these results suggest that the inhibition of RelB nuclear translocation suppresses the tumorigenesis and radioresistance of prostate cancer. In the present study, siRNA RelB efficiently enhanced the radiosensitivity of aggressive prostate cancer cells at low ionizing radiation doses with a limited effect on the growth of normal prostate cells, providing a potential therapeutic approach for the treatment of prostate cancer with high Gleason scores. In line with the observations of others, this study demonstrated that the RM-1 cell survival fraction number and other survival parameters following ionizing radiation treatment were lower in siRNA RelB RM-1 cells compared with those of siRNA control RM-1 cells (Fig. 5). Furthermore, the apoptotic rate was much higher in the siRNA RelB+RT group compared with that of the siRNA control+RT group (Fig. 4).

The blockage of RelB expression by siRNA RelB in the radiotherapy of prostate cancer is capable of inhibiting RelB expression in the cytosol and nucleus. Treatment with siRNA RelB also decreased the RelB DNA binding capacity and augmented the apoptotic rate of RM-1 cells. The clonogenic survival assay revealed that it significantly enhanced the sensitization of prostate cancer to radiotherapy. Overall, the findings from this study provide a foundation for possible future study of radioresistance development.

The inhibition of the NF- $\kappa \mathrm{B}$ alternative pathway via RelB silencing may provide a unique opportunity for selective sensitization of prostate cancer to radiotherapy.

\section{Acknowledgements}

This study was supported by the National Natural Science Foundation of China (grant no. 30671993), the Hubei Provincial Natural Science Foundation of China (grant no. 2011CDB483) and the Fundamental Research Funds for the Central Universities (grant no. 201130202020010).

\section{References}

1. Brawley OW: Prostate cancer epidemiology in the United States. World J Urol 30: 195-200, 2012.

2. Shao Q, Ouyang J, Fan Y, et al: Prostate cancer in the senior men from rural areas in east district of China: contemporary management and 5-year outcomes at multi-institutional collaboration. Cancer Lett 315: 170-177, 2012.

3. Fitzgerald TJ, Wang T, Goel HL, et al: Prostate carcinoma and radiation therapy: therapeutic treatment resistance and strategies for targeted therapeutic intervention. Expert Rev Anticancer Ther 8: 967-974, 2008.

4. Criswell T, Leskov K, Miyamoto S, et al: Transcription factors activated in mammalian cells after clinically relevant doses of ionizing radiation. Oncogene 22: 5813-5827, 2003.

5. Seo SI, Song SY, Kang MR, et al: Immunohistochemical analysis of NF-kappaB signaling proteins IKKepsilon, p50/p105, p52/p100 and RelA in prostate cancers. APMIS 117: 623-628, 2009.

6. Perkins ND and Gilmore TD: Good cop, bad cop: the different faces of NF-кB. Cell Death Differ 13: 759-772, 2006.

7. Enzler T, Sano Y, Choo MK, et al: Cell-selective inhibition of $\mathrm{NF}-\kappa \mathrm{B}$ signaling improves therapeutic index in a melanoma chemotherapy model. Cancer Discov 1: 496-507, 2011.

8. Umezawa K: Possible role of peritoneal NF- $\mathrm{kB}$ in peripheral inflammation and cancer: lessons from the inhibitor DHMEQ. Biomed Pharmacother 65: 252-259, 2011. 
9. Lessard L, Begin LR, Gleave ME, et al: Nuclear localisation of nuclear factor-kappaB transcription factors in prostate cancer: an immunohistochemical study. Br J Cancer 93: 1019-1023, 2005.

10. Murley JS, Kataoka Y and Cao D: Delayed radioprotection by NFkappaB-mediated induction of Sod2 (MnSOD) in SA-NH tumor cells after exposure to clinically used thiol-containing drugs. Radiat Res 162: 536-546, 2004.

11. Xu Y, Josson S, Fang F, Oberley TD, et al: RelB enhances prostate cancer growth: implications for the role of the nuclear factor-kappaB alternative pathway in tumorigenicity. Cancer Res 69: 3267-3271, 2009.

12. Heng-cheng TT, Liu XH, Kuang YU, et al: Construction and identification of mouse RelB siRNA-expressing lentiviral vectors. Sci Res Essays 6: 777-783, 2011.

13. Chendil D, Das A, Dey D, et al: Par-4, a pro-apoptotic gene, inhibits radiation-induced NF kappa B activity and Bcl-2 expression leading to induction of radiosensitivity in human prostate cancer cells PC-3. Cancer Biol Ther 1: 152-160, 2002.

14. Okera M, Bae K, Bernstein E, et al: Evaluation of nuclear factor kappaB and chemokine receptor CXCR4 co-expression in patients with prostate cancer in the Radiation Therapy Oncology Group (RTOG) 8610. BJU Int 108: E51-E58, 2011.

15. Holley AK, Xu Y, St Clair DK and St Clair WH: RelB regulates manganese superoxide dismutase gene and resistance to ionizing radiation of prostate cancer cells. Ann N Y Acad Sci 1201: $129-136,2010$

16. Hanahan D and Weinberg RA: Hallmarks of cancer: the next generation. Cell 144: 646-674, 2011.

17. Watson C, Miller DA, Chin-Sinex H, et al: Suppression of NF-kappaB activity by parthenolide induces X-ray sensitivity through inhibition of split-dose repair in TP53 null prostate cancer cells. Radiat Res 171: 389-396, 2009.

18. Bischoff P, Altmeyer A and Dumont F: Radiosensitising agents for the radiotherapy of cancer: advances in traditional and hypoxia targeted radiosensitisers. Expert Opin Ther Pat 19: 643-662, 2009

19. Carlson DJ, Yenice KM and Orton CG: Tumor hypoxia is an important mechanism of radioresistance in hypofractionated radiotherapy and must be considered in the treatment planning process. Med Phys 38: 6347-6350, 2011.

20. Yacoub A, Miller A, Caron RW, et al: Radiotherapy-induced signal transduction. Endocr Relat Cancer 13: S99-S114, 2006.
21. Koterba K, Beckman MJ and Gewirtz DA: A switch between cytoprotective and cytotoxic autophagy in the radiosensitization of breast tumor cells by chloroquine and vitamin D. Horm Cancer 2: 272-285, 2011.

22. Klokov D, Criswell T, Leskov KS, Araki S, Mayo L and Boothman DA: IR-inducible clusterin gene expression: a protein with potential roles in ionizing radiation-induced adaptive responses, genomic instability, and bystander effects. Mutat Res 568: 97-110, 2004

23. Simon EL, Goel HL, Teider N, et al: High dose fractionated ionizing radiation inhibits prostate cancer cell adhesion and beta(1) integrin expression. Prostate 64: 83-91, 2005.

24. Tang CH and Tsai CC: CCL2 increases MMP-9 expression and cell motility in human chondrosarcoma cells via the Ras/Raf/MEK/ERK/NF- $\kappa$ B signaling pathway. Biochem Pharmacol 83: 335-344, 2012.

25. Zhu L, Zhu B, Yang L, Zhao X, Jiang H and Ma F: RelB regulates $\mathrm{Bcl}-\mathrm{xl}$ expression and the irradiation-induced apoptosis of murine prostate cancer cells. Biomed Rep 3: 354-358, 2014.

26. Umemura N, Zhu J, Mburu YK, et al: Defective NF-кB signaling in metastatic head and neck cancer cells leads to enhanced apoptosis by double-stranded RNA. Cancer Res 72: 45-55, 2012.

27. Pradhan M, Baumgarten SC, Bembinster LA, et al: CBP mediates NF- $\mathrm{kB}$-dependent histone acetylation and estrogen receptor recruitment to an estrogen response element in the BIRC3 promoter. Mol Cell Biol 32: 569-575, 2012.

28. Josson S, Xu Y, Fang F, et al: RelB regulates manganese superoxide dismutase gene and resistance to ionizing radiation of prostate cancer cells. Oncogene 25: 1554-1559, 2006.

29. Xu Y, Fang F, St Clair DK, et al: Suppression of RelB-mediated manganese superoxide dismutase expression reveals a primary mechanism for radiosensitization effect of 1alpha,25-dihydroxyvitamin $\mathrm{D}(3)$ in prostate cancer cells. Mol Cancer Ther 6: 2048-2056, 2007.

30. Xu Y, Fang F, St Clair DK, et al: SN52, a novel nuclear factor-kappaB inhibitor, blocks nuclear import of RelB:p52 dimer and sensitizes prostate cancer cells to ionizing radiation. Mol Cancer Ther 7: 2367-2376, 2008.

31. Reuter S, Charlet J, Juncker T, et al: Effect of curcumin on nuclear factor kappaB signaling pathways in human chronic myelogenous K562 leukemia cells. Ann N Y Acad Sci 1171: 436-447, 2009 